SPECIAL ARTICLE-LEPRA PRIZE ESSAY 1982*

\title{
Leprosy and the Eye
}

\section{T ROGERS}

If asked what they knew about leprosy, few doctors would recall how much the eye is affected by this disease. Yet ocular complication is an extremely common finding in leprosy. If left untreated total blindness is the inevitable result which, in the words of ffytche, ${ }^{11}$ 'adds immeasurably to the already great burdens that these unfortunate individuals carry because of their peripheral nerve lesions, and frequently the onset of severe visual impairment accelerates the complications of this chronic disabling disease'. This essay, therefore, attempts to produce an overview of 'Leprosy and the Eye', describing the prevalence of ocular complications, the complications themselves and some aspects of their treatment.

'Leprosy is a chronic infectious disease primarily affecting the peripheral nervous system and secondarily involving the skin, the mucosa of mouth and upper respiratory tract, the reticuloendothelial system, eyes, bones and testes. ${ }^{21}$ It is caused by the bacterium Mycobacterium leprae and has been estimated to affect from 12 to 15 or even 18 million people throughout the world. The bacteria have been shown to remain viable from nasal blows for several days but the actual root of infection remains a mystery. Whatever this is, in endemic regions a high proportion of the population come into contact with the bacillus. However, $95 \%$ of these mount a normal immune response and do not, therefore, develop leprosy. Of the remaining $5 \%$ some are unable to mount any response to the bacteria and so develop lepromatous leprosy. Others develop a hypersensitivity reaction similar to that found in tuberculosis which is, therefore, called tuberculoid leprosy. These are two polar forms of the disease but a full classification of the disease, put forward by Ridley and Jopling, ${ }^{19}$ includes intermediate forms of leprosy which demonstrate characteristics of both groups. These are Borderline Lepromatous, Mid-Borderline and Borderline Tuberculoid-though in the Madras classification they are all grouped together as Borderline Leprosy. In addition, sub-polar and neuritic types are described as well as an indeterminate. This last group covers many fresh cases which cannot be classified. Fortunately for many patients this phase may be the only manifestation of the disease and they proceed to healing. But for others it may also, after months, pass into one of the more determinate types of leprosy.

It is evident from this classification that the features of leprosy are very variable according to the type of disease suffered. Moreover, changes in the individual's immune state may result in downgrading or upgrading of the disease and, therefore, add to the variability of the features produced.

* This essay was one of the prize-winning entries for 1982 in a yearly essay competition, organized by LEPRA and offered to undergraduates in all the medical schools of the UK. Prize-winning essays for previous years have been published in this journal and in the International Journal of Leprosy. EDITOR. 
In the early stages of lepromatous leprosy macular, papular or nodular skin lesions are produced. These tend to be hypo- or anaesthetic and may even ulcerate. Macular lesions are generally confined to the trunk, whilst nodular lesions may be found on the limbs and face. As will be seen, spread from these lesions may include the adnexae of the eye or onto the globe itself. Involvement of the nerves does occur but is a relatively late feature of lepromatous leprosy. Depending upon which are involved there may be a sensory or motor dysfunction. Because of the widespread nature of the disease anaesthesia tends to be bilateral with a glove-and-stocking distribution. Unnoticed and repeated painless trauma of the hands and feet, therefore, occurs and ultimately results in ulceration, infection and eventually tissue destruction. Motor nerve involvement, however, produces weakness, wasting and deformity making avoidance and care of trauma all the more difficult. If these were not enough the problems of the untreated patient are compounded further by atrophy and absorption of the small bones of the hands and feet which together with severe eye lesions add cruelly to the suffering of these patients.

In tuberculoid leprosy lesions are confined to nerves and the overlying skin. The nerves are grossly thickened and easily palpable, producing sensory or motor dysfunction as in lepromatous leprosy. However, the lesions tend to be unilateral and are not infrequently confined to only one nerve. Anaesthesia and wasting and paralysis is, therefore, more local and deformity less severe. In addition bone atrophy and primary lesions of the eye do not occur which together with better treatment control make the prognosis for these patients better than that of the lepromatous group.

Borderline patients will have features characteristic of both groups, tending one way or the other according to their level of resistance. As a group, however, neurological symptoms tend to develop earlier and more acutely than in either of the polar forms of the disease.

Most authors would agree that the eye is frequently involved in leprosy. Actual figures, however, vary enormously from $1.5 \%{ }^{1}$ to $96 \%{ }^{2}$. On examination of the papers reasons for these large differences become apparent and relate either to patterns and features of the disease and its distribution or to the protocol of the studies involved. Much of the variation attributable to the disease arises from the fact that ocular complications are more common in the lepromatous form of the disease. Therefore, any variation in the ratio of the lepromatous to tuberculoid patients will be reflected in the overall prevalence of ocular complications. This does, indeed, occur and is itself dependent on both racial and climatic factors such that lepromatous disease is more common in Chinese and Mongolian races and in more temperate climates. In addition, the prevalence of ocular complications is dependent on age, the duration of the disease and the efficiency of its control. Variation of the figures may also be attributed to differences in the protocols of the various studies. Such differences included the population under study (i.e. whether from hospital, the community or leprosaria) and the expertise of and equipment available to the author. For example, an ophthalmologist using more sophisticated equipment such as a slit-lamp microscope in contrast to a leprologist using a simple loupe and torch. Additional variation then arises from what is considered to be an ocular complication, if so, is it of leprous origin and then is it potentially sight-threatening?

Nevertheless, despite these differences and the difficulty of comparison, much information can be gained from these studies, a selection of which will now be discussed.

In a study ${ }^{3}$ of 100 patients the high prevalence of facial nerve paralysis was investigated $-49 \%$, of which $26 \%$ were total and $23 \%$ involved the zygomatic branch only. Interestingly $92 \%$ of the cases were of lepromatous patients, so clearly facial nerve damage can also be a common complication of this type of the disease. The study suggested that the cold winters of Baba Baghi were directly responsible for the paralysis of the facial nerve, particularly the superficial branches which have 'been conditioned for damage by the invasion and inflammation set up by M. leprae'. However, facial nerve damage is normally considered to be secondary to the bacterial invasion itself and not primarily as a result of factors such as the cold. Nevertheless, the high prevalence of this complication at Baba Baghi warrants further investigation. It may be, for example, that the cold 
allows greater bacterial proliferation and therefore facilitates the development of this particular complication.

A trial ${ }^{4}$ of 466 Nepalese patients from a leprosarium was detailed and included comparisons between age, sex and duration of the disease, as well as the usual comparisons between lepromatous, borderline and tuberculoid patients. He found $90 \%$ of lepromatous and $65 \%$ of tuberculoid had ocular complications, but included all ocular pathology. Of the $12.7 \%$ of eyes which were blind $4.3 \%$ could be attributed to leprosy. In addition, ocular complications were more frequent in men and this difference was much more significant in the lepromatous form of the disease where there was a 2:1 ratio. Not surprisingly most ocular complications were more common in the older patients and those with longer standing disease.

Statistics produced in this study ${ }^{4}$ are detailed and the differences between the groups quite clear. The actual figures are in fact rather high, but may be accounted for when it is considered that all ocular complications had been included, that $71 \%$ of the patients were older than 40 years and that $66 \%$ had had the disease for more than 20 years.

Two studies were performed at Port Moresby, Papua New Guinea, just 1 year apart. They are interesting because they were carried out on virtually the same population and, although the aims and protocol of each was slightly different, they produced remarkably similar results, except, that is, when considering potentially sight-threatening lesions of which a prevalence of $12 \%{ }^{5}$ and $23 \%{ }^{6}$ were found. These differences could be accounted for because one study ${ }^{5}$ only considered lagophthalmos and iritis to be potentially sight-threatening lesions whilst the other ${ }^{6}$ included in addition keratitis and bilateral corneal anaesthesia, the prevalence of these accounting for the difference. Using his definition Dethlefs ${ }^{5}$ found no potentially sight-threatening lesions in tuberculoid patients and the cases of lagophthalmos were restricted to the borderline group only. Dethlefs compares his overall prevalence of ocular complications, at $52 \%$, with those of another study ${ }^{7}$ who found a prevalence of $72 \%$. The difference is attributed to the difference in the mean ages (26 years ${ }^{5} ; 55$ years $\left.{ }^{7}\right)$. This suggests that the disease may be detected much earlier at Port Moresby, so enabling early treatment. If this is the case it is, therefore, not surprising that there are no potentially sight-threatening lesions in the tuberculoid patients since their disease is more amenable to treatment and more easily cured. Whilst for the lepromatous and borderline patients involvement of the eye may occur early and is more difficult to treat.

In addition to these findings ${ }^{5}$ significant differences for age and the duration of treatment were found $^{6}$, and it was noted that the finding of lagophthalmos only in borderline patients agreed with previous findings that significant nerve lesions occur early in this form of disease.

These two studies on essentially the same population of leprosy sufferers are useful, particularly because the younger age of the patients involved enables comparison with other older groups, and shows that on the whole serious eye lesions are less common in the younger population. More interesting still is the finding that in this younger population eye lesions were most common in the borderline group, and that the lesions were all neuritic in origin. Together with a low prevalence of serious ocular complications it is noteworthy that there were no cases of blindness (except three due to trauma). $\operatorname{Re}^{6}$ suggests that this may be a reflection of the better care and supervision available at Port Moresby, but also notes that it may merely indicate that no blind patients attend the clinic. If this does reflect the results of better supervision and early treatment it is comforting to note the complete absence of blindness and should make those involved in this field all the more keen to detect these unfortunate individuals early and treat and screen for ocular lesions as soon as and as regularly as possible.

A study ${ }^{8}$ was conducted on 430 'mostly fresh' patients attending a leprosy clinic. A prevalence of $25 \%$ ocular complications was found which is compared with the $6.3 \%$ noted by others ${ }^{1}$ who studied 532 patients in Southern Malawi. A somewhat surprising finding was that a high percentage of patients developed eye lesions within 2 years $(70 \%$ at 1 year: $83 \%$ at 2 years) and so it was concluded $^{8}$ that 'the duration of the disease seems to play little role in ocular involvement'. 
Conversely, a direct relationship between ocular affection and the duration of the disease was found ${ }^{1}$. This apparent contradiction can be understood if it is noted that Sehgal ${ }^{1}$ included all ocular complications (and hence also his high prevalence) whilst Ticho \& Ben Sira omitted adnexaeal lesions. These lesions tend to occur in the early stages of the disease and, therefore, it is quite probable that their inclusion in Sehgal's ${ }^{8}$ figures accounts for the difference. Even excluding this Ticho \& Ben Sira's ${ }^{1}$ results are surprisingly low, which was considered to be 'due in part to the wide use of sulphones'. If this is the case, then this adds more weight to Ree's ${ }^{6}$ conclusion that early treatment reduces the prevalence of ocular complications. In addition, Sehgal ${ }^{8}$ considered the possibility of a leprous conjunctivitis since $M$. leprae were found in the smears of 7 out of 8 lepromatous patients with conjunctivitis. Most authors, however, consider conjunctivitis to be due to secondary infection. Since $M$. leprae is found in most bodily secretions, including the tears, of lepromatous patients, their presence in conjunctival smears does not prove a causal relationship with the conjunctivitis of these patients. The possibility of a leprous conjunctivitis should not, however, be dismissed and may form the basis of further study.

A study ${ }^{9}$ of 150 patients with ocular complications found a high percentage of males $(85 \%)$ and the majority of patients over 40 years of age. Binocular involvement occurred in $78 \%$ of patients and there was a high prevalence of blindness $(65 \%)$. This study shows quite clearly the prevalence of ocular complication in the different parts of the eye, demonstrating that affection of the adnexae occurs most commonly. Nevertheless, corneal and uveal tract involvement are also common and more important because they have the potential to cause blindness. Indeed, in a study of blindness in leprosy, ${ }^{11}$ it was found that these two complications accounted for $59 \%$ of the blind eyes. In Das' study, ${ }^{9}$ however, cataracts accounted for $38 \%$ of blind eyes, $32 \%$ of which were mature senile cataracts and only $6 \%$ of which were considered to be secondary to an uveitis.

$\mathrm{T} \mathrm{J}$ ffytche $\mathrm{e}^{11-14}$ has sought to deal with the problem of cataracts, which although not necessarily caused by the leprosy, can nonetheless have devastating consequences on the often severely disabled patients encountered in his trials. These trials on patients from South Korea produced encouraging results with an improvement of vision in all but three patients (out of 44). He also noted that the mean age of patients requiring surgery was lower than non-leprosy patients suggesting that there may be a stronger relationship between leprosy and the development of cataracts than had previously been supposed.

In a second paper ffytche ${ }^{13}$ has considered the pathological processes involved in the uveal changes of leprosy. He produces evidence to support the idea that these are secondary to a neuroparalytic iritis and not due to a primary myositis, as had previously been thought. It was noted that the temperature of the iris is some $3 \cdot 5^{\circ} \mathrm{C}$ centigrade below that of the body so making the small unmyelinated autonomic nerves of the iris a prime target for $M$. leprae. He also suggests that just as 'corneal beads' indicate lepromata of the corneal nerves, so 'iris pearls' represent lepromata of the autonomic nerves of the iris. ffytche presents histological, pharmacological and clinical data to support this theory and whilst accepting that there is as yet no absolute proof he believes that the evidence for a neuroparalytic iritis is strong. This, then, would explain the continuing development of ocular complications in those patients who had been treated successfully for many years, since 'the late effects of neuroparalysis include the slow atrophy of the iris muscle and associated chronic low-grade inflammatory changes'.

So far little has been said of how the eye is actually affected. The following account is a résumé of many papers and a few books and deals with the involvement of the eye by structures.

Involvement of the facial (VII cranial) nerve produces some degree of facial paralysis. This may affect the whole nerve or commonly the zygomatic branch alone, producing weakness of the orbicularis oculi muscle and thus partially or totally preventing closure of the eyelids and resulting in lagophthalmos with or without ectropion. Unable to close his eyes the patient is at risk of developing exposure keratitis and secondary corneal scarring. However, many patients are saved from this because of the maintenance of a good Bell's phenomenon, a good tear film and some 
residual blink reflex. A few are treated surgically with either a medial or lateral tarsorrhaphy whilst fewer still have been given a 'temporalis transfer'. This enables voluntary closure of the lids by clenching the teeth. Some authors believe that facial nerve involvement is not the only cause of lagophthalmos in leprosy, but that a primary leprous myositis, rigidity of the lids or extensive atrophy of the skin and orbicularis oculi muscle may also be responsible for this complication.

Involvement of the trigeminal (Vth cranial) nerve produces hypaesthesia or anaesthesia of the cornea, which also results in an increased risk of incurring corneal trauma and ulceration with consequent corneal scarring and blindness. The not infrequent involvement of both thesenervescan obviously have disastrous sequelae. Direct involvement of either or both is more common in the tuberculoid and borderline forms of the disease. In lepromatous patients involvement is usually a late complication of long-standing disease, but may also result from the acute inflammation accompanying a lepra reaction. Conservative treatment with steroids and atropine may help the latter case.

Adnexaeal involvement is very much more common in lepromatous patients, loss of eyebrows and eyelashes, or madarosis being the most frequent ocular complication recorded. When it occurs the outer third of the eyebrows tends to be affected first and the lower lid before the upper. Hypertrophy and oedema are also common, and nodule formation may produce ectropion and trichiasis, though this complication may be the result of intercurrent trachoma. Epiphora occurs secondary to ectropion, irritation or due to lacrimal duct obstruction. This may occur with primary bacterial invasion or secondary to a dacryocystitis produced by nasal involvement. Nevertheless, despite frequent nasal involvement, florid dacryocystitis is a rare complication.

Conjunctivitis is not an uncommon finding in any form of the disease. It is usually considered to be due to secondary infection which rarely may accompany the aforementioned dacryocystitis but there is also the suggestion that a primary leprous conjunctivitis does occur.

Involvement of the globe itself may also result from direct bacterial invasion of the tissues, but this only occurs in the lepromatous and borderline groups. In the sclera this may result in the formation of lepra nodules or as a more diffuse episcleritis. If severe enough this may develop into a scleritis with secondary atrophy, and staphyloma formation resulting in blindness.

As well as being involved through exposure, the cornea may also be involved by direct spread producing at its simplest corneal nerve opacification and beading. This may progress to pannus or a superficial stromal keratitis and then to an interstitial or even deep keratitis. These last two may result in scarring with or without vascularization and so impede vision, yet the earlier lesions have little affect on vision and usually pass completely unnoticed. Rarely, lepromata may occur in the centre of the cornea with obvious visual defect. This complication has been reported more commonly in South America and Japan, siggesting a regional occurrence. More rarely still a corneal staphyloma may occur which results in blindness.

The uveal tract is frequently involved, but the affects are almost totally confined to its anterior end because of the cooler more suitable environment. A chronic plastic iridocyclitis is the rule but an acute iritis may complicate a lepra reaction or follow the initiation of treatment. Hence previous ocular involvement per se is not necessary for the development of this complication. Evidence of such an acute reaction may be found in the presence of flare, cells, a hypopyon or by the presence of keratic precipitates. Secondary glacoma or cataract may complicate the acute attack but most authors agree that these are not features of the chronic form. Indeed, on the contrary, it has been found ${ }^{15}$ that the intra-ocular pressure of leprosy patients with chronic plastic iridocyclitis was significantly lower than in other leprosy patients. It is probably a consequence of reduced aqueous production secondary to bacterial invasion of the ciliary body. Eventually this will lead to collapse of the globe and, associated with destruction of the tissue, phthisis bulbi will result. Although most authors do not believe that complicated cataracts follow a chronic iridocyclitis, ffytche suggests ${ }^{14}$ that there is a factor which leads to the earlier formation of cataracts and this may be due to the chronic iridocyclitis. Anterior uveitis commonly produces posterior synechiae and iris atrophy. 
Some authors believe this to be a primary myositis; but some others ${ }^{12-14,16}$ believe this to be secondary to neurotrophic changes, as has already been mentioned. Patchy atrophy may result in an acquired coloboma of the iris which is accentuated by the irregularity produced by the posterior synechiae. If severe enough it may result in the formation of 'many pupils', or polycoria. Accompanying the atrophy, debris and exudates are formed, which may collect around the pupil margin as floccules or, if sufficient, may result in total occlusion, 'occlusio pupillae' and blindness. Miosis is also a feature of chronic iridocyclitis, initially resulting from autonomic nerve involvement and later from the consequent preferential atrophy of the dilator muscle. Together with the formation of posterior synechiae this may result in seclusion of the pupil, 'seclusio pupillae', also producing blindness.

Cataracts are a common cause of blindness in leprosy patients. The majority are simple mature senile cataracts but as has been discussed complicated cataracts may follow an acute iritis. Whether or not they complicate the chronic plastic iridocyclitis of leprosy, however, still has to be evaluated.

Involvement of structures posterior to the lens is more debatable though a posterior uveitis has been reported, as well as nodular and macular lesions. Even so these are usually confined to the periphery of the eye and represent extensive ocular involvement.

The best treatment for most of these complications is the removal of the cause, $M$. leprae. In the past this was not possible but the advent of the sulphones in the form of promin (1943), and then dapsone, produced some hope. For a long while dapsone has been used alone but in more recent years dapsone resistance has become a major problem. This, therefore, formed the major part of the discussions of the WHO study group on 'Chemotherapy of leprosy for control programmes' ${ }^{17}$ As a result the study group recommended that dapsone monotherapy be abolished and its use with only one other drug is not advised because of the risk of developing resistance to both drugs if dapsone-resistance $M$. leprae are present. For this reason multidrug regimens have been recommended and include the use of rifampicin with dapsone and clofazimine or if the latter is not acceptable, because of the development of red/blue skin pigmentation, then either prothionamide or ethionamide are acceptable alternatives. This regimen is suggested for multibacillary cases because of the greater likelihood of developing resistance. However, for paucibacillary cases where this risk is minimal rifampicin therapy alone should suffice. However, the addition of dapsone has been recommended by the study group in order to avoid the emergence of rifampicin resistance in those who have been wrongly diagnosed as paucibacillary. Indeed, such resistance has already been described ${ }^{18}$ in two lepromatous patients who were treated with rifampicin monotherapy.

Specific treatment for eye complications includes plastic surgery for madarosis or trichiasis, or in the latter case epilation or electrolysis of the hairs may be effective. Reduced tear secretion may be treated medically with artificial tears, whilst problems with drainage are better treated surgically to establish a new drainage system. If the cause is chronic dacryocystitis, a dacryocystectomy is indicated. Acute and sub-acute infections are better treated with antibiotics and irrigation of the lacrimal sac. Paralysis of the ocular muscles may also be treated with artifical tears or some protective device, such as glasses or goggles. Active exercises and education into voluntary blinking for those with some residual function has been suggested, whilst tarsorrhaphy may be a more lasting solution for those who have not. On the whole infiltrative disease is best treated with anti-leprosy therapy, though individual lesions may require excision. Finally, an acute inflammatory reaction may occur in either the infiltrated eye or as a consequence of circulating degradation products following treatment. In the past therapy was immediately stopped but now it is advocated that anti-leprous therapy should be continued. If mild, simple analgesics and a warm compress may be sufficient to reverse the inflammation and relieve the pain. If more severe, then mydriatic and cycloplegic agents will be required to prevent the production of synechiae whilst the inflammation is treated with NSAID or steroids. If the eye alone is involved drops or ointments can be used but more generalized effects should be treated with systemic steroids. Secondary glaucoma may rarely occur and this is best treated in the conventional manner with acetazolamide whilst anti-inflamma- 
tory medication is continued. If severe enough, however, this too may require surgery in the form of an emergency iridectomy.

What then has so far been accomplished?

The actual ocular complications have now been widely studied and certain features attributed to the different forms of leprosy. Allied to this it is now recognized that there is a marked regional variation in these ocular complications according to race, climate, type of disease, sex, age and duration of the disease. Certain studies have demonstrated that early effective treatment of the disease as a whole can prevent the development of ocular complications, whilst others have shown encouraging results in the management of symptoms or the treatment of intercurrent disease such as cataract. More also is known about the pathology of ocular involvement so that many of the complications can now be explained rather than simply witnessed and documented. In this respect the significance of the chronic plastic iridocyclitis seen in so many lepromatous patients is now better understood and the importance of early treatment to prevent further neural damage better appreciated. Finally, in the words of ffytche: ${ }^{1}$

Visual problems in patients with leprosy very of ten have an effect that far outweighs their severity in normal individuals. The life of the leprosy patient is of necessity one of constant care and attention to the anaesthetic areas of the body in order to avoid trauma and infection; and visual surveillance of the extremities becomes a code to be practised daily by sufferers of this disease. Even a minor abrasion or wound may lead to eventual loss of a digit or permanent impairment of the function of a limb. Blindness if it occurs for any reason adds immeasurably to the already great burdens that these unfortunate individuals carry because of their peripheral nerve lesions, and frequently the onset of visual impairment accelerates the complications of this chronic disabling disease.

And then as Reddy ${ }^{20}$ concludes:

In the early stages the involvement of eye tissues may not produce any symptoms or inconvenience to the patient. However, if they are left untreated they may lead to serious complications. It is therefore essential that in all patients suffering from leprosy, the eyes should be examined carefully and periodically even though the patient does not complain of any eye symptoms. By doing so, the eye complications can be detected in their early stages and their timely treatment will prevent the serious complications and blindness.

\section{Acknowledgments}

My sincere thanks are due to the staff of St Thomas's Hospital Medical School Library who have given me invaluable assistance in collecting journals for this essay.

\section{References}

1 Ticho V, Ben Sira I. Ocular leprosy in Malawi. Br J Ophthalmol, 1970; 53: 107-12.

${ }^{2}$ Harrell JD. Ocular leprosy in the Canal Zone. Int J Lepr, 1977 Jan-Mar; 45 (1): 56-60.

${ }^{3}$ Ramanujam K, Sundar PR, and Khanrei AA. Ocular leprosy in Iran: findings of a random survey at Baba Baghi Leprosarium, Tabriz. Lepr Rev, 1978 Sep; 49 (3): 231-9.

${ }^{4}$ Malla OK, Brandt F, and Artei JAF. Ocular findings in leprosy patients in an institution in Nepal (Khokana). Br J Ophthalmol, 1981; 65: 226-30.

5 Dethlefs R. Prevalence of ocular manifestations of leprosy in Port Moresby, Papua New Guinea. Br J Ophthalmol, 1981 Apr; 65 (4): 223-5. 
${ }^{6}$ Ree GH. Ocular leprosy in Papua New Guinea. Papua New Guinea Med J, 1980 Dec; 23 (4): $182-5$.

7 Shields JA, Waring GO and Marte LA. Ocular findings in leprosy. Am J Ophthalmol, 1974; 77: $880-90$

${ }^{8}$ Sehgal VN, Aggarwal DP and Sehgal N. Ocular leprosy. Indian J Med Res, 1976 Nov; 64 (11): 1600-6.

9 Das R, Aoswani A, Mitna AK and Roy IS. Ocular complications in leprosy. J Indian Med Assoc, 1980 Jan; 74 (1): 5-8.

10 Radhakrishman N and Sathia Albert. Blindness due to leprosy. Indian J Ophthalmol, 1980 Apr: 28 (1): 19-21.

11 ffytche TJ. Cataract surgery in leprosy patients. $J$ R Soc Med, 1979 Nov; 72 (ii): 826-30.

12 ffytche TJ. The eye and leprosy. Lepr Rev, 1981 Jan; 52 (2): 111-19.

13 ffytche TJ. Role of iris changes as a cause of blindness in lepromatous leprosy. Br J Ophthalmol, 1981; 65: 231-9.

14 ffytche TJ. Cataract surgery in the management of the late complications of lepromatous leprosy in South Korea. Br J Ophthalmol, 1981; 65: 243-8.

15 Brandt $\mathrm{F}$ and Malla $\mathrm{OK}$. Ocular finding in leprous patients. A report of a survey in Malunga/Nepal. Albrecht Von Graefes Arch Klin exp Ophthalmol, 1981; 217 (1): 27-34.

16 Slem G. Clinical studies of ocular leprosy. Am J Ophthalmol, 1971; 71: 431-4.

17 WHO Study Group. Chemotherapy of leprosy for control programmes. Technical reports series 675. WHO: Geneva, 1982.

18 Jacobson R R and Hastings RC. Rifampicin-resistant leprosy. Lancet, 1976; ii: 304.

19 Ridley DS and Jopling WH Classification of Leprosy according to immunity, a five group system. Int. J. Lepr. 1966; 34: 255-273

20 Reddy SC, Raja BD and Achary NRSB. Survey of Eye Complications in leprosy in Prakasan District (Andhra Pradesh). Lepr in India. Apr 1981; 53(2): 231-9.

${ }^{21}$ Jopling WH. Handbook of Leprosy (1971). Heinemann, London, P.1. 